A. Hewish

Cavendish Laboratory, Cambridge, England.

\title{
ABSTRACT
}

In this review my aim is to mention very briefly the present state of knowledge over a wide area in order to provide some focus for the wealth of information that this symposium will bring forth. During 13 years a great deal has been discovered, but the jigsaw puzzle of the pulsar model is still in pieces. Some of these have intriguing shapes and seem to fit together, but they do not yet indicate what the picture will be.

\section{THE PULSAR POPULATION}

Surveys of the northern and southern skies have now revealed at least 328 pulsars. Distances estimated from dispersion measures, assuming a simple model for the mean electron density, and making allowance for known HII regions, show that most pulsars lie within a few kpc of the sun. The surface density of the galactic disk increases within a radius of $10 \mathrm{kpc}$ and reaches a maximum at about $6 \mathrm{kpc}$ similar to the distribution of supernova remnants. Pulsars are concentrated towards the galactic plane with a scale height of about $400 \mathrm{pc}$.

Accurate positional measurements, using interferometry and pulse-timing, have enabled proper motions of the order $10-50 \mathrm{~m}$ arcsec $\mathrm{y}^{-1}$ to be obtained. When combined with known distances these lead to typical spatial velocities of the order $180 \mathrm{~km} \mathrm{~s}^{-1}$ with vectors directed away from the plane as expected. Assuming an average $z$-component velocity of $\sim 100 \mathrm{~km} \mathrm{~s}^{-1}$ pulsar 1 ifetimes cannot exceed about $4 \times 10^{6}$ y without conflict with the observed scale height of $400 \mathrm{pc}$. This is significantly shorter than lifetimes derived from spindown in many cases and implies that the stellar magnetic field decreases with time - a feature that is also required to account for the $\mathrm{P}-\dot{\mathrm{P}}$ distribution as discussed later.

The radio luminosity distribution appears to be flattening at the lowest luminosities so that the overall population can be roughly estimated. The total count, assuming circular symmetry and also that 
four pulsars in five are unobserved on account of beaming is $(5 \pm 2) \times 10^{5}$. This population requires a birthrate of about one pulsar in $10 \mathrm{y}$, and is uncomfortably large in comparison to the observed birthrate of supernovae in our galaxy. It is clearly important to refine the parameters involved in the total count in view of its implications for the progenitors of neutron stars.

Only two pulsars have been found in binaries. These have binary periods of $7^{\mathrm{h}} 45^{\mathrm{m}}$ (the famous short period pulsar of great interest in general relativity) and 1265 day.

\section{PULSE TIMING}

Observed pulse periods 1 ie in the range $0.03<\mathrm{P}<4.3 \mathrm{~s}$. The distribution, which cannot be seriously affected by selection, rises steeply to a peak at about $0.5 \mathrm{~s}$ and has a median $\sim 0.8 \mathrm{~s}$. Values of the spindown $\stackrel{P}{\mathrm{P}}$ are known for at least 226 pulsars and span a wide range $10^{-18}<\dot{\mathrm{P}}<10^{-12} \mathrm{~s} \mathrm{~s}^{-1}$ with a median $\sim 3 \mathrm{x} 10^{-15} \mathrm{~s}^{-1}$. A meaningful second derivative $\ddot{\mathrm{P}}$ has been obtained only for the Crab pulsar. In other cases timing irregularities are large enough to obscure second derivatives. Sudden decreases of period (glitches) have been found in 4 pulsars. Vela ( 4 jumps, $\Delta P / P \sim 2 \times 10^{-6}$ ), PSR $1641-45$ and PSR 1325-43 (1 jump each, $\Delta P / P \sim 10^{-7}$ ), Crab pulsar (2 jumps, $\Delta P / P \sim 10^{-8}-10^{-9}$ ). The $\mathrm{Crab}$ and Vela are young pulsars in which jumps are expected to occur more frequently. The limited statistics of the present sample suggest that typical older pulsars undergo roughly 1 jump in $50 \mathrm{y}$.

For the Crab pulsar jumps in period can be understood in terms of starquakes in a rigid crust-superfluid core model. The larger jumps in Vela have been discussed in relation to rigid core models but difficulties have led to more complex ideas concerned with the crustal pinning of quantised vortex lines in a superfluid interior.

Data for 37 pulsars timed over 8 years show some instances where timing residuals, after allowing for $\dot{\mathrm{P}}$, fall within the observational uncertainty of $\sim 0.2 \mathrm{~ms}$, corresponding to a stability of 1 in $10^{12}$. This is close to the limit where scintillation data indicate that interstellar density variations may cause problems unless corrected for by dual-frequency measurements. In many other cases pulsars exhibit residuals, larger than the observational uncertainty, which vary randomly from year to year. The physical significance of these is not yet clear but they should contain useful structural or environmental information concerning the neutron star.

The distribution of $\dot{\mathrm{P}}$, plotted against $\mathrm{P}$, shows an interesting $\mathrm{V}-$ shape which has been interpreted as temporal decay of the neutron star magnetic field. On dipole models of magnetic braking the surface field strength $\mathrm{B}$ is such that $\mathrm{B}^{2} \propto \mathrm{P} \dot{\mathrm{P}}$, and the pulsar age $\propto \mathrm{P} / \dot{\mathrm{P}}$. Plotting $\mathrm{P} \dot{\mathrm{P}}$ against $\mathrm{P} / \dot{\mathrm{P}}$ the evolutionary tracks of individual pulsars would follow horizontal lines if $B$ remained constant. The tracks would curve steeply downwards if $B$ decreased with age and the observed dis- 
tribution can be fitted quite well if B decreases exponentially on a timescale of $0.2 \times 10^{6} \mathrm{y}$. The distribution also suggests that pulsars must cease to radiate when their evolutionary tracks cross some boundary line. The relation $\mathrm{PP}^{-11 / 4} \sim 10^{-15}$, which is the threshold predicted for electron-positron pair production, is a reasonable fit. That pulsars spending a significant fraction of time in a 'nulled' state have points near this line gives added weight to the concept of abrupt extinction at the boundary line.

\section{LUMINOSITIES AND SPECTRA}

Pulsars have radio spectra of the form $\nu^{-\alpha}$ where, typically, $1<\alpha<2$. Low frequency turnovers sometimes occur for $\nu<1 \mathrm{GHz}$ and spectra often steepen for $\nu>1 \mathrm{GHz}$. Radio energy accounts for but a small fraction of the spindown energy $I \Omega \dot{\Omega}$ of the neutron star, typical fractions being $10^{-3}$ to $10^{-8}$. The Crab and Vela pulsars have even smaller efficiencies at radio frequency and emit most of the pulsed energy in $\gamma$-rays at efficiencies of $\sim 10^{-2}$ to $10^{-3}$. These two sources also emit optical pulses at efficiencies of $\sim 10^{-5}$ ( Crab) and $10^{-9}$ (Vela). Unlike the radio spectrum, where pulsars show large intensity variations on many timescales, the optical - $\gamma$ radiation is extremely steady. Over 7 years one component of the optical pulse of the Crab may have decreased by about one per cent. The high frequency spectra lie well above the extrapolated radio spectrum indicating that different radiation regimes must be involved.

\section{PULSE CHARACTERISTICS}

The time-averaged pulse shapes across the whole spectrum make an interesting comparison for the Crab and Vela pulsars. The close synchronism of the main pulse components in the Crab is not repeated for Vela, but the bridge of emission across the pulse centre is a shared feature of the optical and $\gamma$-ray pulses in both. As in other pulsars which display widely spaced main pulse components, it is debatable whether the two peaks are due to a single broad beam with high intensity at its edges, or to a pair of narrow beams pointing in nearly opposite directions. In pulsars as a whole the histogram of component separation extends continuously out to $180^{\circ}$, with no tendency to rise at $180^{\circ}$.

At radio wavelengths pulsars display a rich variety of behaviour on many timescales, but the average pulse profiles remain constant. The overal? pulse-width is about $10^{\circ}$ in longitude. About $25 \%$ of the sources exhibit double-peaked, or more complex profiles, complexity becoming more common at larger values of $\mathrm{P}$; the peak separation (in longitude) tends to increase as $\mathrm{P}$ decreases. The separation is also frequency dependent, varying as $v^{-0.25}$ for $v<1 \mathrm{GHz}$ and remaining almost constant for $\nu \gtrsim 1 \mathrm{GHz}$.

Individual pulses are strongly polarized, usually linearly, with a systematic swing of position angle varying most rapidly at the pulse centre. This is in good agreement with the polar beam (rotating 
vector) model. The simple pattern persists, even when the pulse profile is highly complex, but it is often masked by the presence of two radiation modes having nearly orthogonal position angles. Each mode, on its own, follows the simple pattern but one or other of the modes dominates over specific ranges of longitude. Strict orthogonality is not always observed and modes separated by $65^{\circ}-90^{\circ}$ in position angle are found. Circular polarization, when present, often maximises at the pulse centre or reverses its sense there. This again agrees with the polar beam model. The degree of polarization usually decreases at higher frequencies and can vary by $\gtrsim 2$ between $100-430 \mathrm{MHz}$.

Within individual pulses the intensity is modulated on various timescales, the relative importance of which changes markedly across the radio spectrum. The subpulse regime has a timescale $\sim 5 \times 10^{-3} \mathrm{P}$ and the microstructure a scale of $\lesssim 5 \times 10^{-4} \mathrm{P}$. The correlation of both with $\mathrm{P}$ is approximate but significant. Microstructure is occasionally quasi-periodic $\left(Q \stackrel{<}{\curvearrowright}\right.$ ) with period $\sim 10^{-3} \mathrm{P}$. Subpulses often exhibit a systematic drift in longitude, from pulse to pulse, which modulates the intensity at a given longitude with period $\mathrm{P}_{3}$. The drift rate can be highly regular or variable. Usually $\mathrm{P}_{3} \sim$ a few times $P$, but values up to $40 P$ have been observed. Intensity variations due to microstructure and drifting subpulses are mainly low frequency phenomena and decrease rapidly above about $1 \mathrm{GHz}$. By contrast, the integrated pulse intensity shows increasing pulse to pulse variations at frequencies above $1 \mathrm{GHz}$.

Two further important effects are nulling and mode-switching. Nulling is an abrupt cessation of radiation at all frequencies which typically lasts for a few seconds but may endure for hours. Pulsars with substantial nulling ( $>5 \%$ of total time in null state) have $\dot{\mathrm{PP}}^{-11 / 4} \sim 10^{-15}$ and may be near some radiation threshold. Modeswitching is an abrupt change of pulse profile which may also entail changes in the subpulse and drifting behaviour.

Drifting, nulling and mode-switching are distinctive phenomena which must severely constrain any theory of pulsar radiation. Especially dramatic and clear-cut cases deserve close attention. For example, PSR 0809+74 shows a remarkable connection between nulling and drifting which would be hard to discern in a less regular drifter. At low frequencies this source emits all its radiation in drifting subpulses for which $P_{3}=11 \mathrm{P}$ with $\mathrm{Q} \gtrsim 500$ (an observational 1imit). Nulls are quite frequent and the first pulse to appear at the end of a null exhibits subpulses at the same longitudes that they occupied when the radiation ceased, although the subpulses can be located anywhere within the pulse window. The subpulses 'remember' where they were for up to $13 \mathrm{~s}$ (the longest null observed). Immediately following a null the drift rate is slightly slower and takes a few seconds to recover its normal rate. Observations made simultaneously at 102 and $1720 \mathrm{MHz}$ have shown that the drift rate at $1720 \mathrm{MHz}$ is almost twice as slow as at $102 \mathrm{MHz}$ although $\mathrm{P}_{3}$ remains the same. The subpulses at each frequency appear to be physically related since their intensity 
variations are significantly correlated, even though their instantaneous longitudes are usually different.

Another notable pulsar is PSR $1237+25$ which has a 5-component profile with approximate mirror symmetry about its centre. The two outermost components are strongly modulated, in antiphase, with $\mathrm{P}_{3}=2.8 \mathrm{P}$, while the three inner components show no periodic modulation. In terms of the polar beam model three separate conical beam systems appear to be involved. A system of excitation zones spiralling inwards has also been suggested. Finally PSR 1822-09 deserves mention on account of its intriguing connection between modeswitching and drifting subpulses. In one radiation mode two subpulses are present, one of which shows occasional drifting with $\mathrm{P}_{3}=11 \mathrm{P}$; in the second mode one subpulse vanishes and the other shows well-defined drifting with $\mathrm{P}_{3}=40 \mathrm{P}$.

\section{PULSAR MODELS}

Rapidly spinning neutron stars are believed to be surrounded by a corotating magnetosphere in regions where magnetic field 1 ines form closed loops inside the velocity of light cylinder. The local charge density is assumed to adjust itself until $\underline{E} . \underline{B}=0$ in the rotating frame, hence generating a density $\propto \underline{\Omega} . \underline{B}$. This assumes that charges of either sign can be drawn freely from the surface and that there are no difficulties in populating field lines along which the charge density changes sign. 'Open' field lines, which intersect the light cylinder, are taken to be regions where $\underline{E} \cdot \underline{B} \neq 0$ and along which charges may suffer large acceleration. Whether charges leave the star through the light cylinder or lose energy via radiation and return to the star along different field 1 ines is under debate. In the former case a stable flow requires charge balance and the difficulty arises that open field 1 ines are preferentially associated with charges of one sign only.

Until these atmospheric problems have been solved it may be rash to consider detailed radiation mechanisms. At present most attention is given to models in which the radio emission is generated not far above the polar caps, at heights of less than about $10^{3} \mathrm{~km}$. A substantial fraction of the voltage arising from unipolar induction across the polar cap is assumed to be available for accelerating charges along open field lines close to the stellar surface. This could be the case when $\mathrm{E}$ is not strong enough to draw + ions from the star in the vicinity of the polar cap and if $\Omega . B<0$. An electron in this region accelerates violently towards the surface, its spiral motion along $B$ generating $\gamma^{-}$ radiation which subsequently initiates electron-positron pairs. Repetition of this sequence generates a particle-cascade, positrons moving outwards and electrons inwards. Local heating of the surface due to electron bombardment could be sufficient to release ions by thermionic emission, and their subsequent interaction with thermal X-rays from the hot-spot could generate further pairs, the final result being a sustained electron-positron discharge. Beyond the acceleration zone a relativistic outflow of electron-positron plasma (Lorentz factor $\lesssim 200$ ) 
might be penetrated by both an ion stream (Lorentz factor $\sim 500$ ) and a positron stream (Lorentz factor $\sim 10^{6}$ ). The motion of ions through the $\mathrm{e}^{+-}$plasma could generate two-stream instabilities rapidly enough for charge-bunching to occur, finally leading to the emission of radio waves by coherent curvature radiation. The radiation would be emitted tangentially to $B$ on open field lines at distances around $10^{2}-$ $10^{3} \mathrm{~km}$ from the surface.

It has also been suggested that radiation could be generated close to the 1 ight cylinder, which has a radius of $\sim 10^{5} \mathrm{~km}$ for a typical pulsar, but no detailed physical models have been outlined. No direct observational evidence can yet determine the site of the radiation zone. For polar-cap models involving narrow-band coherence the radiation at higher frequencies is emitted lower down. This would lead to a temporal retardation factor $\Delta t \sim \Delta R / C$ where $\Delta R$ is the difference in height of the radiation zones at two frequencies. If the charge bunches corotate aberration would also occur, but there seems to be no reason why the relativistic outflow should partake in corotation. Observations of pulses at different frequencies have not. shown retardation so that any separation of the sites of high and low frequency radio emission must be less than about $100 \mathrm{~km}$.

On general grounds it is hard to see how the complex behaviour associated with mode-switching and drifting subpulses (including the memory effect) could be sustained near the light cylinder. At large distances there are also problems in maintaining a constant polarization profile when the radiation field may exceed the stellar magnetic field. The polar-cap model appears to provide a suitable environment to explain the polarization and pulse profiles. The increase in width of the profiles at lower frequencies and drifting subpulses (in particular PSR $0809+74$ observed at widely different frequencies) lend support to the idea of streaming along field lines which generates different frequencies at different heights. On the other hand the mechanism of drifting is hard to understand. Plasma drift in ExB fields has been suggested, and the thermal time constant of hotspots on the surface has been invoked to explain the drifting subpulse memory. If the revival of discharges after a null is due to thermionic emission of ions from hotspots it seems that the time constant must be chosen with care. It must be long enough to survive nulls of up to $13 \mathrm{~s}$, but short enough to avoid initiating a discharge around the entire annulus under normal drifting, when the circulating hotspots travel a distance equal to their separation in about the same time. 